RESEARCH AND PRACTICE

\title{
The need for culturally-tailored smartphone applications for weight control
}

\author{
Steven S. Coughlin, PhD, $\mathrm{MPH}^{1,2}$, Dale Hardy, PhD, RD, LD, CDE, CHES ${ }^{3}$, Lee S. Caplan, MD, $\mathrm{PhD}^{4}$ \\ ${ }^{1}$ Department of Community Health and Sustainability, Division of Public Health, University of Massachusetts, Lowell, MA; ${ }^{2}$ Department of \\ Epidemiology, Rollins School of Public Health, Emory University, Atlanta, GA; ${ }^{3}$ College of Allied Health Sciences, Augusta University, \\ Augusta, GA; ${ }^{4}$ Department of Community Health and Preventive Medicine, Morehouse School of Medicine, Atlanta, GA
}

\begin{abstract}
Background: Approximately 35\% of U.S. adults are obese, and this rate is expected to increase by almost $50 \%$ by 2030 . New media such as smartphone applications (apps) provide a useful and low-cost way to disseminate weight control information. For many culturally distinctive population subgroups, however, there is currently an absence of research-tested smartphone apps for weight control.
\end{abstract}

Methods: In this commentary, we highlight the need for culturally tailored smartphone apps for weight control and offer recommendations for further research by providing two specific examples: 1) the characteristic dietary patterns and absence of smartphone apps for weight loss for Hispanic Americans, in English and Spanish, and 2) and need for smartphone apps for weight loss for Brazilian Americans, including those who speak Portuguese.

Results: Smartphone apps can be an effective intervention for improving diet and nutrition, encouraging physical activity, and reducing obesity, but few randomized controlled trials have been conducted of stand-alone smartphone apps for weight loss that focus primarily on self-monitoring of diet and physical activity. Further, there have been no published studies of apps for promoting healthy diet, better nutrition, increasing levels of physical activity, and weight loss among Hispanic Americans or Brazilian Americans.

Conclusions: Low-cost, effective e-Health interventions (healthcare practices supported by electronic processes) are needed to promote physical activity, healthy eating, and weight control in culturally distinctive subgroups of the population. For weight loss, apps should be developed by use of evidence-based approaches that relate to behavioral theories. Additional public health research is needed to identify low-cost, effective strategies for weight loss for people who have varying levels of health literacy, and for non-English speakers. Culturally tailored e-Health interventions for weight control are more likely to address the needs of individuals and increase their motivation to engage in health promoting behaviors.

Key Words: Brazilians; Hispanics; diet; nutrition; obesity; physical activity; smartphones

\section{INTRODUCTION}

The high prevalence of obesity in the U.S. population is a serious threat to the health of Americans because of causal linkages between obesity and cancer of the breast, colon, and other sites; diabetes; cardiovascular diseases; arthritis; and other adverse health consequences (Dietz, W. 2015; Sallis, J \& Glanz, K. 2009). Nutrition, physical activity, and energy balance are important determinants of weight loss and maintenance of healthy weight, which are preventive measures to deter adverse health conditions. However, approximately 35\% of adults in the U.S. are obese (Johnson, N, et al. 2014) and this rate is expected to increase; by the year 2030, the obesity rate among U.S. adults is projected to increase to $51 \%$ (Finkelstein, E, et al.). Currently, 37.5\% of Georgians are obese (CDC).

Established interventions for weight loss through caloric restriction, healthy eating, and physical activity are resource-intensive, a factor that poses barriers for full participation and widespread dissemination. Smartphone applications (apps) provide a useful and low-cost way to disseminate weight control information to the general population and to particular at-risk populations (Coughlin, S, et al. 2015). Smartphone apps can be an effective intervention for improving diet and nutrition, encouraging physical activity, and addressing obesity (Allen, J, et al. 2013; Coughlin, S, et al. 2015). For weight loss, however, few randomized controlled trials have been conducted for stand-alone smartphone apps that focus primarily on selfmonitoring of diet and physical activity (Coughlin, S, et al. 2015; Carter, M, et al. 2013; Allen, J, et al. 2013). In addition, there is currently an absence of research-tested culturally tailored smartphone apps for weight control that are suitable for distinctive population subgroups or for nonEnglish speakers.

A mobile app is a computer program designed to run on smartphones or other mobile devices. All major smartphone platforms provide third-party developers with application programming interfaces that can be used to build special purpose applications referred to as native apps (Bender, J, et al. 2013). Smartphone apps can have a variety of features including visually-engaging designs, video and audio 
capabilities, unrestricted text capabilities, access with or without cellular or Internet connection, content sharable via social media, and tracking progress anywhere and anytime (Bricker, J, et al. 2014). Common techniques include providing feedback, goal-setting, self-monitoring, and planning social support and change (Azar, K, et al. 2013).

In 2013, 58\% of adults in the U.S. owned a smartphone; by 2020, the percentage is projected to surpass $90 \%$ (Bricker, J, et al. 2014; Smith, A. 2013). Currently, about $60 \%$ of Hispanic Americans own a smartphone (Smith, A. 2013). Over half (52\%) of smartphone users seek health information using their smart devices and may utilize health apps to monitor and manage their health.

Although various apps are available from major smartphone platforms (e.g., Android, iPhone, Windows phone, and BlackBerry) for weight management, promoting healthy eating, increasing physical activity, and other healthy behaviors associated with reduced risk of chronic disease, few have been tested for acceptability or effectiveness (Coughlin, S, et al. 2015; Bender, J, et al. 2013; Backinger, C \& Auguston, E. 2011; Coughlin, S. 2014; Bert, F, et al. 2014). Further, few of these apps are based on theories of health behavior change, most do not include evidence-based features such as self-monitoring and reinforcement, and some do not provide evidence-based recommendations for weight loss (Coughlin, S, et al. 2015; Bender, J, et al. 2013).

There are currently no research-tested smartphone apps for weight control for people with varying levels of health literacy and eHealth literacy, or for non-English speakers. For weight loss, such apps should be developed using evidencebased approaches based on behavioral theories. Ideally, app users should be able to select food and drink from a database and log items into an electronic food diary, record physical activity in the diary and receive instant feedback on their energy expenditure and track their progress graphically.

Dietary guidelines for Americans were developed jointly by the U.S. Department of Agriculture (USDA) and the Department of Health and Human Services (HHS) (HHS. 2015). The 20152020 Dietary Guidelines were designed to encourage healthy eating by emphasizing the following: 1) Follow a healthy eating pattern at an appropriate calorie level; 2) Focus on variety, nutrient density, and amount; 3) Limit calories from added sugars and saturated fats and reduce sodium intake; and 4) Shift to healthier food and beverage choices. A healthy eating pattern includes a variety of vegetables from all of the subgroups; fruits; grains (especially whole grains); fat-free or low-fat dairy products; and a variety of protein foods, including seafood, lean meats, poultry, legumes, nuts, seeds, and soy products (HHS. 2015). A healthy eating pattern involves limited consumption of saturated fats and trans fats, added sugars, and sodium (HHS. 2015). The USDA's ChooseMyPlate program provides practical information to help consumers build healthier diets with user-friendly nutrition information in English, Spanish, and other languages (USDA). Culturally tailored healthy recipes and menu suggestions are included.
The HHS 2008 Physical Activity Guidelines for Americans emphasize that all adults should avoid inactivity (HHS. 2008). Some physical activity is better than none, and adults who participate in any amount of physical activity gain health benefits. For substantial health benefits, adults should perform at least 150 minutes a week of moderate-intensity aerobic physical activity, 75 minutes a week of vigorousintensity aerobic physical activity, or an equivalent combination of moderate- and vigorous-intensity aerobic activity. Such activity should be performed in episodes of at least 10 minutes, and, preferably, be spread throughout the week (HHS. 2008).

\section{METHODS \& RESULTS}

Two examples highlight the need for culturally tailored smartphone apps for weight control and offer recommendations for further research: 1) smartphone apps for weight loss for Hispanic Americans, in English and Spanish, and 2) and similar smartphone apps for Brazilian Americans, many of whom speak Portuguese and do not self-identify as Hispanic.

\section{Example One: Smartphone Apps for Weight Control Among Hispanic Americans}

Hispanics are the largest ethnic group in the U.S., currently comprising $17.4 \%$ of the population (US Census). Rates of obesity are higher among Hispanic men and women than among their non-Hispanic White counterparts (Flegal, K, et al. 2012; Haffner, S, et al. 1986). Hispanic individuals with an elevated body mass index (BMI) have a higher prevalence of diabetes, hypertension, high LDL-cholesterol levels, and low HDL-cholesterol levels (Kaplan, R, et al. 2014; Schneiderman, N, et al. 2014; Heiss, G, et al. 2014).

The prevalence of obesity and diabetes varies across Hispanic subgroups (Schneiderman, N, et al. 2014). For Mexican Americans, acculturation is related to a less healthy diet that includes more fat and "fast foods." Epidemiologic studies have shown that general obesity and central obesity are more prevalent among Mexican Americans than nonHispanic Whites (Haffner, S, et al. 1986). Mexican Americans who become adapted to the U.S. culture may adopt a less-healthy diet and increase their risk for cardiovascular diseases (Hazuda, H, et al. 1991). As determined in phase 2 of the San Antonio Heart Study, which included 2,013 Mexican Americans and 928 nonHispanic Whites, aged 25-65 years, the prevalence of overweight was greater for Mexican Americans than for non-Hispanic Whites, and the ethnic difference was greater in women than in men (Hazuda, H, et al. 1991). For Mexican Americans, the effects of socioeconomic status (SES) and assimilation on overall obesity and body fat distribution differed by sex group. For men, SES and cultural assimilation were associated with less favorable body fat distribution. For women, SES and assimilation were associated with lower overall obesity and more favorable body fat distribution. Interventions to reduce obesity among Mexican Americans should take these subgroup differences into account (Hazuda, H, et al. 1991).

In the U.S., Puerto Ricans are the second largest Hispanic subgroup after Mexican Americans. The traditional Puerto 
Rican dietary pattern has been associated with greater BMI and waist circumference (Lin, H, et al. 2003). For Puerto Rican adults in the Greater Boston area, language-based acculturation relates to healthy diet (e.g., increased consumption of whole grains, fruit, and non-starchy vegetables) and less central obesity (Van Rompay, M, et al. 2012). Acculturation, however, is also associated with increased consumption of saturated fat and decreased consumption of legumes. Puerto Rican women in the U.S. have a higher prevalence of the central adiposity-related metabolic syndrome than other Hispanic women (40.9\% vs. 36.0\%) (Van Rompay, M, et al. 2012). Like other adults in the U.S., most Hispanics do not consume the recommended amount of 5 servings daily of fruits and vegetables; many consume a diet low in whole grains and high in red meat, total fat, sugar, and energy-dense nutrient-poor foods (Van Rompay, M, et al. 2012). In Georgia, the median intake of fruits and vegetables are only 1.0 and 1.6 servings daily, respectively (CDC).

As this example illustrates, there are characteristic dietary patterns among Hispanic subgroups. Although published studies indicate that smartphone apps can be an effective intervention for improving diet and nutrition, encouraging increased physical activity, and addressing interventions to decrease the prevalence of obesity, there have been no published studies of apps for promoting a healthy diet, better nutrition, increasing levels of physical activity, and weight loss among Hispanic Americans.

\section{Example Two: Smartphone Apps for Weight Control among Brazilian Americans}

In terms of geographic size and population size, Brazil is the largest country in South America and the fifth largest in the world. The population of Brazil is diverse by race and ethnicity. Of the population, $48.43 \%$ self-identify as White, $43.8 \%$ as Pardo (brown), $6.84 \%$ as Black (Afro-Brazilians), $0.58 \%$ as Asian, and $0.28 \%$ as Amerindian (indigena or indigenous). The Pardo population includes descendants of European Whites, Amerindians, and Afro-Brazilians. Over 371,529 people of Brazilian descent are known to live in the U.S. (US Census), although estimates range up to $1,100,000$.

To an increasing extent, the populations of middle-income countries such as Brazil are transitioning from a traditional diet to a Westernized diet that includes more calories, fat, and obesogenic, ultra-processed food products that have higher energy density, high free sugar contents, and less fiber than natural or minimally processed food (Monteiro, C, et al. 2013). The traditional dietary pattern in Brazil, with a high consumption of staple and basic foods, is associated with a reduced BMI and lower risk of cardiovascular disease (Olinto, M, et al. 2012; Sichieri, R. 2002). Anecdotal information suggests that Brazilians in the U.S. (a) consume less fruit and vegetables than they would in Brazil, where ripe fruit and native vegetables are abundant (personal communication, Dr. Eduardo Siqueira, January 2016) and (b) that many are obese (personal communication, Natalicia Tracy, January 2016).

Although there have been no epidemiologic studies of obesity, diet, and nutrition among Brazilians in the U.S., such studies have been conducted in Brazil. Bezerra et al. (Bezerra, I, et al. 2014) examined differences in adult food group intake by sex and age using data from the 2008-2009 Brazilian Household Budget Survey $(\mathrm{n}=26,390)$ and the 2007-2008 National Health and Nutrition Examination Survey (NHANES) $(n=5,420)$, both of which are based upon probabilistic samples. The objective was to compare food intake of Brazilian adults with American adults to identify possible dietary factors associated with the increase in obesity in Brazil. For the U.S., there were, relative to Brazil, higher frequencies of intake for most food categories, except for meat, rice and rice dishes; beans and legumes; spreads; and coffee and tea. In both countries, young adults (ages 20-39 years) reported greater consumption of meat, poultry and fish mixed dishes; pizza and pasta; and soft drinks relative to older adults (ages $\geq 60$ years). The consumption of deli and cured meats, savory snacks (e.g., potato chips), pizza and pasta, dairy products, soft drinks, and alcohol was higher in the U.S. than in Brazil. Meat, poultry and fish mixed dishes (13\%), breads (11\%), sweets and confections (8\%), pizza and pasta (7\%), and dairy products (6\%) were the top five food category sources of energy intake among American adults, contributing to $44 \%$ of total energy intake. The top five food categories in Brazil were rice and rice dishes (13\%), meat $(11 \%)$, beans and legumes (10\%), breads $(10 \%)$, and coffee and tea (6\%). Traditional plant-based foods, such as rice and beans, were substantial contributors to the Brazilian diet. In a separate analysis of data from the 2008-2009 national survey of individual food intake, Bezerra et al. (Bezerra, I, et al. 2013) examined away-from-home food habits of Brazilians, restricting their analysis to individuals aged 10 years or older who lived in urban areas of Brazil $(n=25,753)$. Of this population, $43 \%$ consumed at least one food item away from home. The consumption of foods away from home was associated with a greater energy intake. The highest away-from-home energy sources were foods with a high content of energy, such as alcoholic beverages (59\%), baked and deep-fried snacks (54\%), pizza (42\%), soft drinks (40\%), sandwiches (40\%), and sweets and desserts (30\%) (Bezerra, I, et al. 2013).

Using data from the 2008-2009 Brazilian Household Budget Survey $(\mathrm{n}=32,898)$, Louzada et al. (2015) examined consumption of ultra-processed foods and the nutritional dietary profile of Brazilians aged 10 years or over. Examples of ultra-processed foods included sweet and salty biscuits, potato chips, sweet pastries and cakes, fast foods, instant noodles, and ready-made meals. The average daily energy consumption per capita was 1,866 kcal, with $69.5 \%$ being provided by natural or minimally processed foods, $9.0 \%$ by processed foods and $21.5 \%$ by ultra-processed food (Louzada, M, et al. 2015).

Martins et al. (2013) examined temporal trends in household food and drink consumption in Brazil using data from Household Budget Surveys conducted in 1987-1988, 19951996, 2002-2003, and 2008-2009. In all of the surveys, probabilistic samples of households in metropolitan areas were studied; the scope for the last two surveys was national. The caloric share of ultra-processed products significantly increased in the Brazilian diet over this time 
period (from 20.8\% to 25.4\%). The increase in the ultraprocessed products occurred across all income quintiles (Martins, A, et al. 2013).

Although data are currently lacking for Brazilians living in the U.S., there are characteristic dietary patterns among Brazilians in Brazil. Smartphone apps can be an effective intervention for improving diet and nutrition and weight loss, but there have been no studies of apps for weight control among Brazilian Americans.

\section{CONCLUSIONS}

Additional research is needed to identify low-cost, effective e-Health interventions for weight loss, accomplished through caloric restriction, healthy eating, and physical activity, that are culturally specific for population subgroups with varying levels of health literacy. Health promotion messages, tailored for subgroups defined by culture, ethnicity, and language spoken in the household, are likely to address the needs of individuals, increase their motivation to engage in health promoting behaviors, be perceived as personally relevant, and lead to a greater likelihood of behavior change.

\section{Acknowledgements}

The authors thank Drs. Eduardo Siqueira, Natalicia Tracy, Katherine Tucker, Herpreet Thind, Benyuan Liu, and Selina Smith for helpful discussions on the use of smartphones to promote healthy eating in diverse populations.

\section{References}

Allen J, Stephens J, Dennison Himmelfarb C, et al. Randomized controlled pilot study testing use of smartphone technology for obesity treatment. J Obes 2013:10.1155/2013/151597 http://dx.doi.org/10.1155/2013/151597

Azar K, Lesser L, Laing B, et al. Mobile applications for weight management: theory-based content analysis. Am J Prev Med 2013;45:583-9.

Backinger C, Augustson E. Where there's an app, there's a way? Am J Prev Med 2011;40:390-1.

Bender J, Yue R, To M, et al. A lot of action, but not in the right direction: systematic review and content analysis of smartphone applications for the prevention, detection, and management of cancer. J Med Internet Res. 2013; 15: e287.

Bert F, Giacometti M, Gualano M, Siliquini R. Smartphones and health promotion: a review of the evidence. J Med Syst 2014;38:995. doi: 10.1007/s10916-013-9995-7.

Bezerra IN, de Moura Souza A, Pereira RA, Sichieri R. Contribution of foods consumed away from home to energy intake in Brazilian urban areas: the 2008-2009 Nationwide Dietary Survey. Br J Nutr 2013;109:1276-83.

Bezerra IN, Goldman J, Rhodes DG, et al. Difference in adults food group intake by sex and age groups comparing Brazil and United States nationwide surveys. Nutrition J 2014;13:74.

Bricker J, Mull K, Kientz J, et al. Randomized, controlled trial of a smartphone app for smoking cessation using acceptance and commitment therapy. Drug Alcohol Depend 2014;143:87-94.

Carter M, Burley V, Nykjaer C, Cade J. Adherence to a smartphone application for weight loss compared to website and paper diary: pilot randomized controlled trial. J Med Internet Res 2013;15:e32.

Centers for Disease Control and Prevention FastStats http://www.cdc.gov/nchs/fastats/

Coughlin S. Intervention approaches for addressing breast cancer disparities among African American women. Ann Trans Med
Epidemiol 2014;1:pii 1001.

http://www.ncbi.nlm.nih.gov/pmc/articles/PMC4283773/

Coughlin S, Whitehead M, Sheats J, et al. Smartphone applications for promoting healthy diet and nutrition: a literature review. Jacobs J Food Nutr 2015

http://www.jacobspublishers.com/images/FoodNutrition/J_J_Fo od_Nutri_2_3_021.pdf

Dietz W. The response of the US Centers for Disease Control and Prevention to the obesity epidemic. Annu Rev Public Health 2015;36:575-96.

Finkelstein E, Khavjou O, Thompson H, et al. Obesity and severe obesity forecasts through 2030. Am J Prev Med 2012;42:56370.

Flegal KM, Carroll MD, Kit BK, Ogden CL. Prevalence of obesity and trends in the distribution of body mass index among US adults, 1999-2010. JAMA 2012;307:491-7.

Haffner S, Stern M, Hazuda H, et al. Upper body and centralized adiposity in Mexican Americans and non-Hispanic whites: relationship to body mass index and other behavioral and demographic variables. Int J Obes 1986;10:493-502.

Hazuda H, Mitchell B, Haffner S, Stern M. Obesity in Mexican American subgroups: findings from the San Antonio Heart Study. Am J Clin Nutr 1991;53(6 Suppl):1529S-1534S.

Heiss G, Snyder M, Teng Y, et al. Prevalence of metabolic syndrome among Hispanics/Latinos of diverse background: The Hispanic Community Health Study/Study of Latinos. Diabetes Care 2014;37:2391-99.

HHS 2008 Physical Activity Guidelines for Americans http://health.gov/paguidelines/

HHS 2015-2020 Dietary Guidelines for Americans http://health.gov/dietaryguidelines/

Johnson N, Hayes L, Brown K. CDC National Health Report: leading causes of morbidity and mortality and associated behavioral risk and protective factors-United States, 20052013. MMWR Surveill Summ 2014;31(63 Suppl 4):3-27.

Kaplan R, Aviles-Santa L, Parrinello C, et al. Body mass index, sex, and cardiovascular disease risk factors among Hispanic/Latino adults: Hispanic Community Health Study/Study of Latinos. J Am Heart Assoc 2014;3:e000923.

Lin H, Bermudez O, Tucker K. Dietary patterns of Hispanic elders are associated with acculturation and obesity. J Nutr 2003;133:3651-7.

Louzada ML, Bortoletto Martins AP, Canella DS, et al. Ultraprocessed foods and the nutritional dietary profile in Brazil. Rev Saude Publica 2015;49-38.

Martins AP, Levy RB, Claro RM, et al. Increased contribution of ultra-processed food products in the Brazilian diet (1987-2009). Rev Saude Publica 2013;47:656-65.

Monteiro CA, Moubarac JC, Cannon G, Ng SW, Popkin B. Ultraprocessed products are becoming dominant in the global food system. Obes Rev 2013;14(Suppl 2):21-8.

Olinto MT, Gigante DP, Horta B, et al. Major dietary patterns and cardiovascular risk factors among young Brazilian adults. Eur J Nutr 2012;51:281-91.

Sallis J, Glanz K. Physical activity and food environments: solutions to the obesity epidemic. Milbank Q 2009;87:123-54.

Schneiderman N, Liabre M, Cowie C, et al. Prevalence of diabetes among Hispanics/Latinos from diverse backgrounds: The Hispanic Community Health Study/Study of Latinos (HCHS/SOL). Diabetes Care 2014;37:2233-9.

Sichieri R. Dietary patterns and their associations with obesity in the Brazilian city of Rio de Janeiro. Obes Res 2002;10:42-8.

Smith A. Smartphone ownership_-2013 update. Available at: http://www.pewinternet.org/2013/06/05/smartphone-ownership2013/

USDA ChooseMyPlate http://www.choosemyplate.gov/

U.S. Census QuickFacts http://quickfacts.census.gov/qfd/states/00000.html 
J Ga Public Health Assoc (2016) Vol 5, No. 3

Van Rompay M, McKeown N, Castaneda-Sceppa C, et al. Acculturation and sociocultural influences on dietary intake and health status among Puerto Rican adults in Massachusetts. J Acad Nutr Diet 2012;112:64-74. 